\title{
Bordes, derivas y sueños: \\ The Bartlett School of Architecture
}

\author{
Borders, drifts and dreams: \\ The Bartlett School of Architecture
}

Chгіstian Beгоs* / CB

<Resumen>

Este artículo trata de indagar sobre la forma de enseñar y aprender a hacer arquitectura en The Bartlet, UCL, por medio de una entrevista

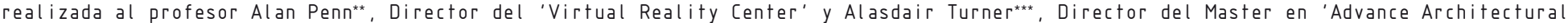
Computing' en Londres, Julio 2006. Como conclusión, se propone una crítica al sistema de enseñanza actual en la Universidad de Chile en base a la comparación con el actual sistema en UCL

$<$ Abstract >

The following article discusses the way architecture is taught and learned at The Bartlett School of Architecture. Alan Penn. the Director of the Virtual Reality Center and Alasdair Turner Director of the Mater in Advanced Architectural Computing are interviewed by the author. Then the author closes the article with a critical analysis of the way Architecture is taught in the Universidad de Chile.

<PALABRAS CLAVE>

LONDRES / BEAUX ART / ESTRUCTURAS DE ENSEÑANZA

KKEY WORDS >

LONDON / BEAUX ART / TEACHING STRUCTURES
"Design has an end purpose: to create a formal presence, to coerce all the intelligent forces towards this presence, to celebrate creativity. To deal primary with things, stuff and by implication, places and experiences».

Peter CoOK ${ }^{1}$

* Arquitecto Universidad de Chile, MSc Advance Architectural Studies, The Bartlett, UCL.

* Alan Penn es arquitecto, investigador y Profesor de arquitectura y Urban Computing en The Bartlett, UCL. ES además el director del Virtual Reality Centre y ha sido uno de los principales desarrolladores de las técnicas de Space Syntax y se aplicaron en una amplia gama de proyectos.

*** Alasdair Turner es Lecturer e investigador del Virtual Reality Centre y dirige el MSc in Advance Architectural Computing en The Bartlett. Su investigación se basa en flujos urbanos e interacción social, análisis espacial, diseño generativo, y redes neuronales de comportamiento humano.

1 Peter Cook es arquitecto, crítico y académico en distintas escuelas a nivel mundial. Su trabajo como diseñador ha sido ampliamente reconocido por medio de Archigram y como académico fue Chairman de The Bartlett desde 1990 donde se destacó por la rehabilitación que indujo en la forma de enseñar y hacer arquitectura. 
En los últimos treinta años la forma de enseñar y ver la arquitectura ha cambiado drásticamente en el Reino Unido y en especial en The Bartlet, la Escuela de Arquitectura de UCL en Londres. Con la llegada de Peter Cook a mediados de los años 80, la Escuela abandona un sistema de enseñanza tradicional, de talleres y cátedras teóricas y toma un perfil orientado hacia el Beaux Art. Incentivando la exploración y la creación en el campo del diseño arquitectónico y creando un sistema educacional basado en las denominadas "Units» donde se concentraba todas las áreas del conocimiento ligadas a la arquitectura.

En un artículo de Neil Spiller², donde se refiere a los límites de la arquitectura contemporánea, explica como el cruzar ciertas barreras puede permitir un trabajo entre disciplinas nunca antes relacionadas con nuestra profesión. Según Spiller la exploración de nuevas áreas, ideas y conceptos pueden no resolver un problema concreto pero ayudarían a generar nuevas preguntas y nuevas incertidumbres. En el caso de ustedes, en los laboratorios de realidad virtual y de Space Syntax, están constantemente cruzando esos bordes y vinculando el mundo científico con el mundo del diseño. ¿Cómo entienden la interacción entre estas áreas a nivel profesional y académico hoy en día?

[Alan]: Creo que uno de los problemas en la interacción entre ambos mundos es un sentimiento que existe en ciertas áreas del diseño arquitectónico donde se cree que el conocimiento puede llegar a contaminar la creatividad, creo que eso es un gran error, pero es comprensible ya que proviene de no entender los diferentes niveles en que el diseño toma lugar. El diseño en sí tiene dos niveles: e primero en el que se desarrolla la generación y creación de la forma; y el segundo, debido a que es un objeto social, donde tienes que visualizar, comprender y evaluar críticamente lo que se ha propuesto, y esto es un proceso cíclico en el que alternas los dos niveles.

Cuando estás bosquejando un proyecto, estás en un constante diálogo con un trozo de papel en un primer nivel, que es intuitivo. Es bastante difícil de separar cuando estás en el segundo nivel, en un rol más analítico. Pero como arquitecto circulas continuamente entre ambos. Es muy fácil para algunos arquitectos decir que es un solo rol, un solo acto de diseñar, pero tan pronto cuando se establece ese error, niegas la entrada al conocimiento objetivo que viene a informar al proceso. Creo que en las escuelas de arquitectura muchos profesores tienen miedo de dar a sus alumnos textos a leer, que los alumnos puedan entender lo que leen, porque temen a la contaminación del acto de diseñar. Pero quizás es como «Species», quizás necesitas uno de ellos en cada escuela.

I CB: Desde la creación de los laboratorios de investigación en The Bartlett, tanto el "Virtual Reality Center» y el "Space Syntax Laboratory» se han introducidos distintas herramientas para asesorar el proceso de diseño. ¿Qué es lo que sucede cuando estas nuevas tecnologías se hacen parte del ejercicio a nivel profesional?

[Alan]: Creo que hay dos líneas o dos posibilidades de lo que puede llegar a pasar cuando introduces un nuevo tipo de conocimiento científico. Uno es la división del trabajo, lo que anteriormente había sido hecho por los profesionales usando su intuición, se traslada a un grupo de profesionales con un conocimiento objetivo que actúan en un grupo de trabajo, cooperando en el proceso junto a otros profesionales y son los arquitectos los que están a cargo de los aspectos intangibles e intuitivos del proceso de diseño. Esto ha pasado a través de los años en el área de la ingeniería, por ejemplo, si volvemos al Renacimiento, habían arquitectos que pasaban años después de diseñar el edificio calculando la estructura. Hoy en día tienes ingenieros estructurales que utilizan distintos software para calcular, y en prácticamente todas las oficinas de arquitectura hay un ingeniero sentado en la mesa de trabajo junto al arquitecto. Space Syntax está en esta relación ahora, las cosas que antes se dejaban puramente a la intuición y a la experiencia, en términos de patrones de comportamiento humano, ahora son calculables y Space Syntax Limited actúa al nivel de los ingenieros alrededor de la mesa de trabajo junto a otros arquitectos. Eso es una parte de la relación, pero no tiene que ser solamente de esa forma, también se puede enseñar a los futuros arquitectos como resolver los problemas que se le presentan, para diseñar y a la vez utilizar las herramientas del conocimiento objetivo. En este caso, entra en juego el software, y como diseñamos el software para ser lo suficientemente sencillo sin tener la necesidad de llamar al ingeniero. Esa es también otra forma de desarrollar el problema.

[Alasdair]: Y eso es exactamente lo que tratamos de hacer en el proyecto "Intelligent Architecture» en 1995. Sin embargo, creo que siempre existe un riesgo cuando el componente social en arquitectura queda entregado a los especialistas.
[Alan]: El riesgo existe, pero ahí esta la diferencia entre hacer un edificio y hacer arquitectura. La arquitectura siempre va a traer devuelta todos sus componentes al proceso de diseño. El proceso constructivo, por ejemplo, ha sido prácticamente internalizado en las oficinas en el Reino Unido, especificando hasta el último detalle y usando herramientas digitales para asistir al diseño del proyecto. De esta forma se minimizan los riesgos que podrían ocurrir en el proceso generativo de la forma. Cuando se diseña se está siempre en el borde de lo posible, creando formas que quizás nunca antes fueron testeadas; el proceso creativo es entonces cautivante, pero riesgoso, riesgo que se asume con el conocimiento científico para evaluar asegurar un resultado correcto y factible. Entonces, la objetividad científica hace el proceso creativo más libre de riesgos.

/ CB: Según tu punto de vista, se necesita un arquitecto que pueda manejar distintas áreas de conocimiento, algunas cada vez más alejadas de las escuelas, como la sociología o la geografía. ¿Cómo educas a un arquitecto para que sea capaz de interactuar entre diversas disciplinas?

[Alan]: Creo que el conocimiento que se es debe proveer debe ser más integral que especifico, para estar preparados a dialogar en distintas áreas. Luego si se requiere una especialización entra en juego la escuela de posgrado. Ahora, que suceda de esta forma o no, esa es otra pregunta.

[Alasdair]: Mi percepción, ya que no soy arquitecto, es que con frecuencia son los mismos arquitectos quienes no dan crédito a otro tipo de conocimiento, creyendo solo en sí mismos y en el área intangible del diseño, más que en la complementariedad con otras áreas. Por lo general las respuestas son... «no ese no es mi trabajo... ese es el trabajo del constructor, del ingeniero, de space syntax, etc.», que están ahí para resolver mi proyecto. Lamentablemente si el arquitecto empieza a hacer eso, pierde todo el contacto con su edificio.

[Alan]: Claro, entonces se convierte en un manejador de riesgos solamente, tratando de cubrir todas las respuestas, entregando toda la responsabilidad a una serie de profesionales. El proyecto de diseño requiere ser responsable en la forma en como se enfrenta; el arquitecto debiera tomar lo mejor de la ciencia, para limitar los riesgos que acarrea su proyecto, pero no debería dejar que esos riesgos limitaran su ambición como creador. La impronta debe ser siempre hacer que las cosas funcionen mejor y hacerlas más bellas, no importando lo que estés haciendo. 
/ CB: Se define entonces, a un arquitecto con una mirada interdisciplinaria que a su vez debe estar encargado del proceso de diseño. ¿Cuáles son las herramientas que este arquitecto necesita para desenvolverse en el mundo profesional?

[Alan]: Bueno, la habilidad básica que se enseña en la escuela de pregrado en The Bartlett es diseñar, es acerca del pensamiento que hay detrás del proceso de diseño. Creo que eso es lo único que nos diferenciaría con otras profesiones en las cuales no se entiende el diseño como lo entendemos nosotros. Por ejemplo, hace poco estuve en un taller con distintos profesionales, en el cual se hicieron grupos de trabajo, algunos tenían arquitectos en ellos y otros no. Se hacía un encargo a todos los equipos que deberían resolver durante el día y el resultado fue que los equipos que no tenían un arquitecto con ellos, no pudieron manejar los límites del encargo y desecharon las restricciones para crear más «libremente». Al final esos equipos fallaron al tratar de generar un resultado. En cambio, aquellos equipos que tenían un arquitecto manejaron mejor las restricciones y las utilizaron para su beneficio haciendo un diseño más acabado. Creo que eso se da porque sin la habilidad que aprendes en la escuela con ciertas restricciones y límites, sería imposible llegar a un nivel de diseño más profundo. Es falso que la innovación viene con la falta de restricciones, de hecho es completamente al revés, pero eso lo aprendes solamente haciendo arquitectura, no se enseña explícitamente sino que tácitamente a través del encargo, a través de la reflexión y la acción. Esa es la idea general atrás de una actividad reflectiva como el diseño.

[Alasdair]: Por otro lado, la escuela de posgrado toma gente que ya viene entrenada en diseño. Lo que esencialmente se les entrega es un set extra de conocimientos que pueden tomar e incluir en sus diseños en el futuro.

Además nos interesa vincular a los alumnos con la investigación, explorando nuevas técnicas en diseño y tratando de entender específicamente como esas técnicas funcionarían para luego ser aplicadas. Sin embargo, es muy tentador aplicar algo nuevo directamente sin saber que lo que se esta haciendo. Por ejemplo, en mi experiencia con nurbs "surfaces», las curvas que se pueden generar pueden ser muy llamativas, una cuerva con varios puntos de control que puedes manipular y que muchos arquitectos utilizan desarrollando arquitectura de «burbujas». El problema es cuando se quiere traducir eso a la realidad y verificar las curvas, ahí aparece el resultado de alguien que no conoce la herramienta que se usó, que solo movió un par de puntos en el software para hacer una forma que se viera bien; inventó una historia a su cliente y lo mostró como su «arquitectura». Creo que ahí está el error, si se quiere usar una nueva herramienta se tiene que entender completamente y se tiene que comprender su potencial como herramienta de investigación.

[Alan]: También tienes que ver de donde vienen los estudiantes a la escuela de posgrado en The Bartlett. La gran mayoría de los alumnos del $\mathrm{AAC}^{3}$ o el $\mathrm{AAS}^{4}$, ya tuvieron antes una práctica como profesionales, pero no se sienten intelectualmente motivados por el ejercicio que estaban llevando a cabo. O piensan que puede haber algo más, planteándose una crítica al sistema de educación de pregrado y una crítica también al sistema profesional que tiene muchas tareas tediosas asociadas. Entonces, volver a la escuela por un período les plantea nuevas exigencias intelectuales. En el caso del Reino Unido los alumnos vienen por un curso de Master y al volver a sus países son los encargados de desarrollar intelectualmente la práctica, modificando la forma de hacer y pensar en nuestra profesión.

/ CB: Bajo ese punto de vista crítico hacia el desarrollo tanto de la profesión como de las escuelas de arquitectura, las escuelas de posgrado toman cada vez mayor fuerza. ¿Cómo se maneja entonces el grado de especialización del arquitecto, si lo que se requiere es un arquitecto más integral?

[Alan]: Ese es uno de los problemas fundamentales del ser arquitecto, debes ser capaz de diseñar y pensar en un proyecto a nivel global e interdisciplinario a la vez, reuniendo además otras profesiones y especialistas. La idea de ser arquitecto especialista en hospitales o vivienda, por ejemplo, trae asociada la pregunta de si diseñar hospitales es diferente a diseñar a nivel urbano, o hasta hacer diseño de interior. Creo que ese nivel de especialidad viene con la práctica y los desarrollas a través de prueba y error. Por otro lado, cuando existe conocimiento científico asociado a la arquitectura, es decir, un conocimiento objetivo aplicado al diseño, comienzas a evitar riesgos y viene la división del trabajo. Entonces, el arquitecto puede dedicarse a su tarea fundamental que es diseñar y a la vez coordinar a los especialistas y grupos de conocimiento asociados a la ciencia. Utilicemos como ejemplo la ingeniería estructural: el arquitecto necesita el conocimiento suficiente para mantener una discusión con el ingeniero, para entender lo que dice y para poder dialogar; lo que necesitas es poder reunir a los especialistas en una discusión. Pero hay que tener cuidado, porque eso es muy distinto a decir que la arquitectura es un campo donde se reúnen los otros especialistas, porque la arquitectura perdería su propia definición, en términos del proceso de diseño, del como diseñar y del hecho que todo interactúa alrededor del espacio en arquitectura.

[Alasdair]: A mí me parece que separar demasiado las áreas de conocimiento es un gran error en las escuelas de arquitectura, como separar las áreas de tecnología con las áreas de vivienda social. Por ejemplo, lo que puede pasar con la compra de última tecnología como las máquinas de prototipado rápido, tú no necesitas la última máquina del mercado para entender lo que tienes que hacer con ellas.

[Alan]: Bueno, en un sentido lo que los alumnos hacen es investigar que es lo que pueden hacer con estas máquinas, explorando los sistemas constructivos que pueden generar, la pregunta es: ¿Se relaciona esto con la arquitectura en un medio real? Pues entonces la respuesta es no, nunca vas a ocupar una máquina de ese tamaño para construir un trozo de arquitectura a escala real y de hecho su uso es particularmente irrelevante y complicado. Pero, por otro lado, lo que ellos están aprendiendo son las relaciones genéricas en un set de posibilidades estéticas y a su vez la tecnología de producción en masa, por supuesto finalmente todos los trabajos se parecen demasiado, debido a las restricciones de la máquina. Ahí comienza el ciclo, luego de un tiempo necesitas comprar una máquina nueva, con nuevas restricciones.

La implantación de tecnologías digitales, definitivamente ha cambiado la forma de ver la arquitectura, pero antes, Peter Cook, uno de los arquitectos británicos más sobresalientes y más transgresores, también había modificado la forma de educar arquitectos en The Bartlett. ¿Cómo fue ese proceso y cuál es la diferencia con el antiguo sistema?

[Alan]: Bueno, en el caso de The Bartlett; la escuela actual es muy distinta de la escuela donde yo estudié antes de que se hiciera cargo Peter Cook, la forma en que se instauró la nueva enseñanza del diseño es radicalmente diferente.

Creo que ha habido logros, pero también pérdidas importantes en la transformación de la enseñanza. A veces quedo sorprendido por la falta de conocimiento básico sobre arquitectura. Los alumnos pasan tanto tiempo dedicados 


\section{wwwimex-cl}

\section{. म . - . . Tus Mejores Herranitentas de Trabajo}

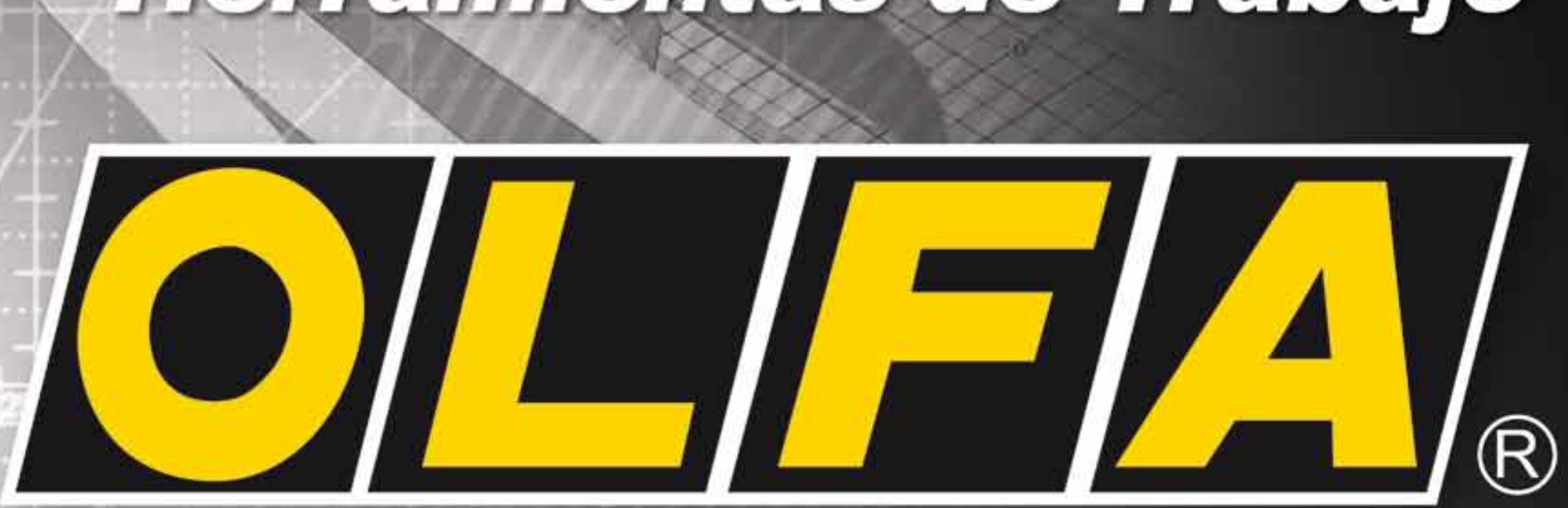

Made in Japan 
al Studio que no dejan tiempo para la historia de la arquitectura, para entender el diseño de detalles y una serie de otras cosas. Creo que el temor a veces es que se especialice demasiado y se pierda la visión del todo.

Para hacer un marco de la época creo que la relación entre arquitectura y «fashion» en este caso es crucial, ya que una serie de estilos o tendencias distintas existieron durante los '60. En esa época la implicancia social de la profesión en la escuela de arquitectura tenia un valor tremendo, hasta el punto en que los estudiantes recibían un encargo de diseño en la escuela y no entregaban un diseño formal sino una corrección del texto que contenía el encargo, es decir, la enseñanza estaba fuertemente influenciada por los movimientos políticos del momento. A mediados de los ' 80 , Cook tomó el control de la escuela siguiendo los pasos que ya había marcado la Architectural Association. Cook reformuló la estructura curricular de The Bartlett imponiendo un sistema de unidades en el pregrado, cada una de ellas a cargo de un Master Unit o tutor que proponía un tema específico a desarrollar durante el año académico, las cátedras fueron prácticamente eliminadas y el proceso de aprendizaje se centró en el proyecto de diseño. Las áreas de urbanismo, estructuras, construcción y teoría fueron asociadas a cada Unit dependiendo de su tema, de los profesores y críticos invitados por el Unit Master. Los departamentos se agruparon en la escuela de posgrado, y su interacción radicó en la investigación en sus áreas especificas y en los vínculos que proponían para con cada una de las unidades.

Aparte del cambio al interior de la escuela ocurrió también un cambio general en la profesión en el Reino Unido, en el cual redefinió la posición del arquitecto en relación a las otras profesiones vinculadas al medio ambiente construido. En dicho cambio participaron una serie de profesiones que tomaron campos que antes pertenecían a nuestra área de conocimiento, como la ingeniería estructural, el manejo de proyectos, el cálculo financiero, entre otros, y se separaron de nuestra disciplina. Entonces ahí cabe la pregunta de ¿Qué es lo que hace el arquitecto? ¿Cuál es su rol?

Si lo analizamos hoy en día, podríamos decir que la arquitectura está volviendo a tomar algunas de las responsabilidades que le competen, retomando temas como la sustentabilidad, el cambio climático, la pobreza, etc. Todos los arquitectos quieren participar de esos temas, porque ahora es de su interés, entonces puedes ver que el «fashion» está cambiando, el arquitecto como un artista no es suficiente, entonces ahora tienes que seguir siendo por un lado el artista, pero ahora con responsabilidad medioambiental y social. Desde mi punto de vista, las tendencias son cíclicas, dependen de un tiempo determinado y los intereses de la industria cambian de acuerdo a esos ciclos.

/ CB: Recién referían a ciertos cambios generales en The Bartlett y en el Reino Unido, Pero, ¿Cómo definirían los cambios a nivel de estructura y funcionamiento al interior de la Facultad?, ¿Cómo es la separación entre los departamentos de investigación y la escuela de pregrado?

[Alan]: Bueno, en general tienes esa separación en un primer nivel en The Bartlett, pero el elemento que las agrupa son las Units, que tienen una filosofía más social en términos de la orientación sobre las problemáticas de la arquitectura, y esa es una de las diferencias fundamentales entre la estructura de unidades y la estructura de un año de carrera profesiona con materias paralelas al Studio. Eso es lo que promovió Peter Cook y es lo que nos permite tener una diversidad de enfoques y al mismo tiempo nos permite seguir y liderar el "fashion sin arriesgar la escuela completa. Así, si un año la tendencia va por el lado de la sustentabilidad, tendremos el próximo año más Units dedicadas a temas de desarrollo sustentable o cambio climático, más profesores trabajando sobre esas materias y apoyando a los estudiantes. No va a pasar, por ejemplo, que todos los studios de pregrado cambian su enfoque a un proyecto de arquitectura sustentable, porque eso sería muy riesgoso desde el punto de vista de la escuela como organización, porque quizás ese enfoque no es el correcto o no es el camino que el "fashion» está tomando. De esta forma, podemos acomodar distintos portafolios, distintos acercamientos a la arquitectura y evaluar como funcionan y si no resultan, se cierran. Así puedes cambiar rápidamente y sólo arriesgas una pequeña parte del grupo de estudiantes y profesores.

[Alasdair]: Los alumnos tienen sólo una Unit durante el año académico, no hay cursos teóricos en paralelo, sólo un curso de teoría asociado y alguno relacionado con tecnología. No hay ninguna cátedra de pizarrón aparte de Historia y Teoría que abarca esa área. Pero en cambio, tienes mucha gente invitada desde el ámbito profesional que viene a asesorar a las Units dependiendo de los temas que están desarrollando. Por ejemplo ARUP, está en este minuto trabajando con un taller, guiando procesos y sistemas estructurales con profesionales de primer nivel al interior de una Unit específica. Entonces, nuevamente tienes el contacto directo con el mundo profesional. Y en el caso de tu Universidad, ¿cómo es la estructura de la facultad en Chile?
/ CB: La estructura sigue siendo en general la misma de cuando egresé, un taller por año o por semestre dedicado al diseño, y una serie de cátedras paralelas divididas en urbanismo, estructuras, construcción, etcétera.

[Alan]: Es como el caso de la estructura social antigua al interior de la facultad en los '60, donde había constructores en un lado, planificadores en el otro, etc. En el caso de la estructura de Peter Cook hay varios Unit Masters, y cada uno de ellos con distintos backgrounds y distintos acercamientos a la arquitectura, pero todos las áreas están involucradas en el diseño y todos están educando a un arquitecto integral. Esto está basado en la teoría de formación pedagógica de Bernstein en «Frame and Classiffication». En el sistema que comentas, tienes una clasificación del grupo de materias que pueden involucrar la enseñanza de la arquitectura, tienes un área social, un área de construcción, un área urbana, etc. Es un sistema altamente clasificado, a cada una corresponde a un grupo de individuos, estos grupos dan distintas cátedras y los estudiantes están a cargo de sintetizar los conocimientos entre un curso y otro. En el caso de The Bartlett tienes distintas unidades que parecen mecánicamente similares entre ellas, pero ellas se diferencian en las temáticas, y cada una trae consigo a los especialistas que necesita para desarrollar su enfoque y agrupar todos los conocimientos. El problema del esquema que tú comentas es que pierdes la solidaridad entre el grupo social del staff.

[Alasdair]: En ese sistema los departamentos de investigación se separan de lo que está pasando en la escuela de pregrado. Investigación y enseñanza no van en conjunto para aportar lo que genera la facultad como un todo y la estructura general pierde fuerza.

[Alan]: Es difícil decir si el sistema de The Bartlett es mejor o peor, porque se ha movido entre un sistema y otro a lo largo del tiempo. Creo que ambos tienen deficiencias y posibilidades también.

En general, el problema es que se separan esos cursos paralelos de lo que es el diseño, estás diciendo: estos cursos no son diseño, son otra cosa y por eso son externos al taller. Lo que se hace en The Bartlett es decir no, estas clases son todas partes de un todo, son todas partes del proceso de diseño y la Unit es el motor del todo. Entonces el taller es el que llama a las distintas partes a incorporarse, dependiendo del tema que están cada una desarrollando y de cómo piensa enseñar a diseñar. Son dos modelos fundamentalmente opuestos. 